\title{
Microstructural Alteration in Conventional Metallic Coatings by Carbonaceous Additives (Graphene Oxide and Carbon Nanotubes)
}

\author{
Rekha M Y ${ }^{1}$, Abhay Gupta ${ }^{2}$, Kompella Jyotheender ${ }^{3}$ and Chandan Srivastava ${ }^{4}$ \\ ${ }^{1}$ Metallurgical and Materials Engineering, University of Alabama, Tuscaloosa, AL, USA, Tuscaloosa, \\ Alabama, United States, ${ }^{2}$ IISc Bangalore, Karnataka, India, ${ }^{3}$ IISc-Bangalore, Bengaluru, Karnataka, \\ India, ${ }^{4}$ IISc Bangalore, Bengaluru, Karnataka, India
}

Coatings have been traditionally used to protect the underlying substrate against corrosion. In recent years, it has been shown that incorporation of carbonaceous additives such as graphene, graphene oxide and carbon nanotubes (CNTs) can produce significant changes in the electrochemical behaviour of the composite coatings, and for an optimum volume fraction of the additive, significantly large reduction in the corrosion current density can be achieved [1,2]. It has been proposed that decrease in the corrosion rate in the case of graphene and graphene oxide is primarily due to their impermeability to the passage of the corrosive fluid, whereas in the case of CNT, the effect is mainly due to its hydrophobicity. Although the sensitivity of the corrosion response of the composite coatings with minor addition of the carbonaceous materials is well established, there still exists a debate about the beneficial or detrimental effect of such addition. This debate is mainly due to the fact that most of the studies on these composite coatings essentially focus on the electrochemical properties without exploring the microstructure-property correlations [3]. Our work on understanding the correlation between the microstructure and the corrosion behaviour of metallic coatings containing carbonaceous additives has illustrated that at the "optimum" concentration of the additives, distinct and "favourable" changes in the coating microstructure concerning texture, grain boundary constitution, gain size, compositional partitioning and phase evolution happen which compliments the intrinsic protective properties of the additive to synergistically impart significantly higher corrosion resistance performance to the composite coatings. This paper illustrates three interesting examples.

In the first example, SnNi and SnNi-graphene oxide composite coatings were electrodeposited over mild steel substrate. Pristine SnNi coating contained Sn and Sn-Ni solid solution grains (compositional map in Fig. 1(a)). Incorporation of graphene oxide produced nearly pure $\mathrm{Sn}$ grains with $\mathrm{Ni}$ occupying the grain boundary regions (compositional map in Fig. 1(b)). This alteration in microstructure led to reduction in the corrosion current density $\left(\mathrm{i}_{\text {corr }}\right)$ value from $16.2 \mu \mathrm{A} / \mathrm{cm}^{2}$ for $\mathrm{SnNi}$ coating to 4.6 for $\mathrm{SnNi}$-graphene oxide composite coating. In the second example, $\mathrm{SnBi}$ and $\mathrm{SnBi}$-graphene oxide coatings were electrodeposited over mild steel substrate. Corrosion resistance of coatings increased with the incorporation of graphene oxide ( $\mathrm{i}_{\text {corr }}$ value of $1.76 \mu \mathrm{A} / \mathrm{cm}^{2}$ and $0.57 \mu \mathrm{A} / \mathrm{cm}^{2}$ for $\mathrm{SnBi}$ and $\mathrm{SnBi}$-graphene oxide coatings respectively). The coating matrix consisted of Sn and Bi-rich phases (Fig. 1(c-f). Incorporation of graphene oxide enriched Sn-rich phase because of high adsorption affinity of Bi towards graphene oxide sheets (Fig. 1(g-j)). Electron backscatter diffraction (EBSD) technique showed that the coating with the graphene oxide contained highest fraction of low energy low angle grain boundaries and grew along the low index planes for Sn-rich grains which promoted higher corrosion resistance. In the third example, pristine $\mathrm{Ni}$ coatings and $\mathrm{Ni}-\mathrm{CNT}$ composite coatings were electrodeposited over mild steel substrate. Incorporation of CNTs significantly enhanced the corrosion resistance performance of the coatings ( $i_{\text {corr }}$ value of $10.6 \mu \mathrm{A} / \mathrm{cm}^{2}$ and $0.66 \mu \mathrm{A} / \mathrm{cm}^{2}$ for $\mathrm{Ni}$ and Ni-CNT coatings respectively). EBSD measurements (Fig. 2(a)) illustrated that the CNT addition promoted columnar grain growth with (011) texture along the coating growth direction (Fig. 2(b)). CNT incorporation promoted the formation of low angle grain boundaries (LAGBs) and coincidence site lattices (CSLs) (Fig. 2(c,d)) thereby, enhancing the corrosion resistance performance of the coatings. 


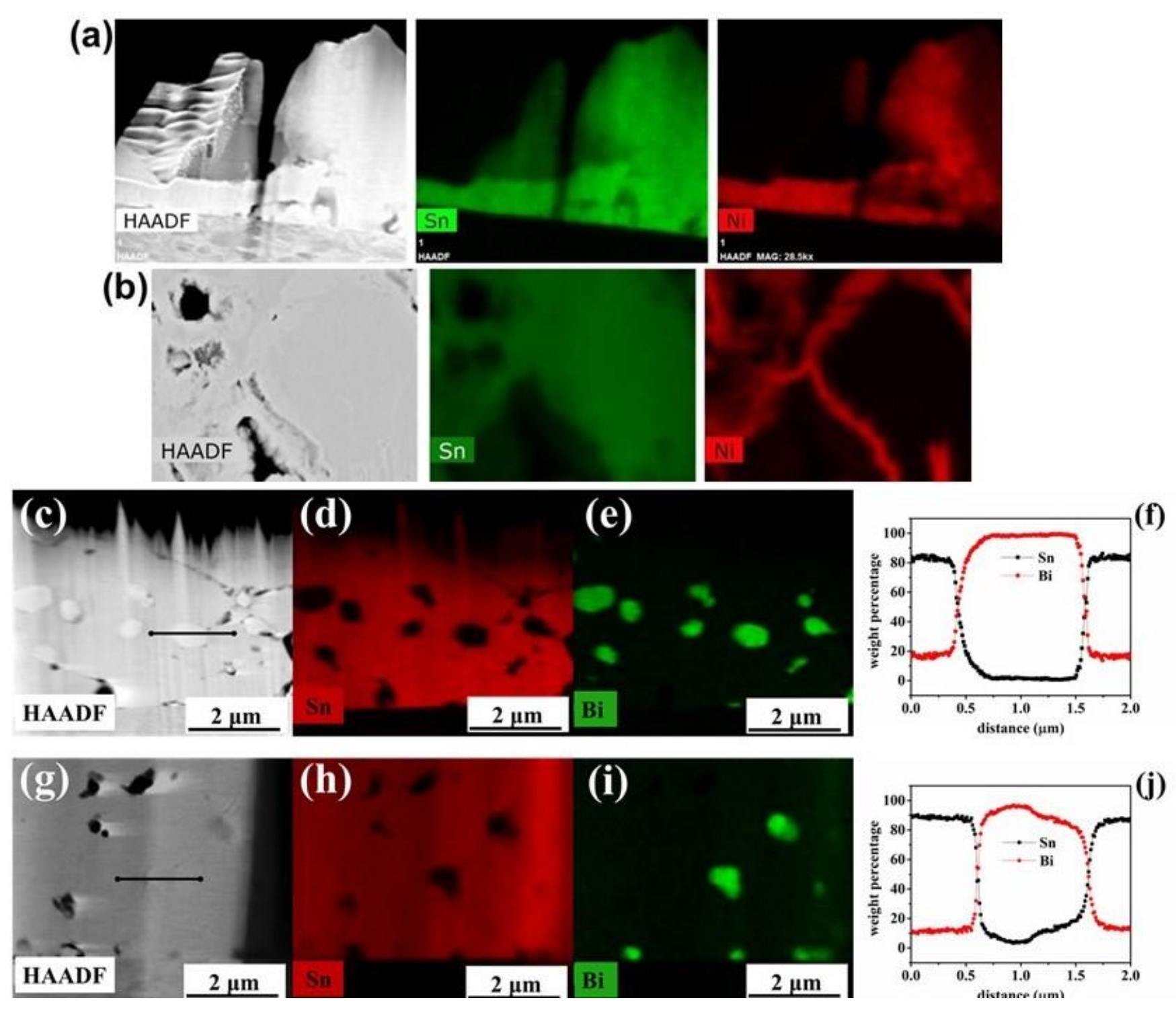

Figure 1. Compositional maps of (a) SnNi and (b) SnNi-graphene oxide composite coatings, (c) STEM micrograph, (d) Sn map, (e) Bi map from SnBi coating.(f) compositional scan along the line indicated in (c). (g) STEM micrograph, (h) Sn map, (i) Bi map from SnBi-graphene oxide coating.(j) compositional scan along the line indicated in $(\mathrm{g})$. 

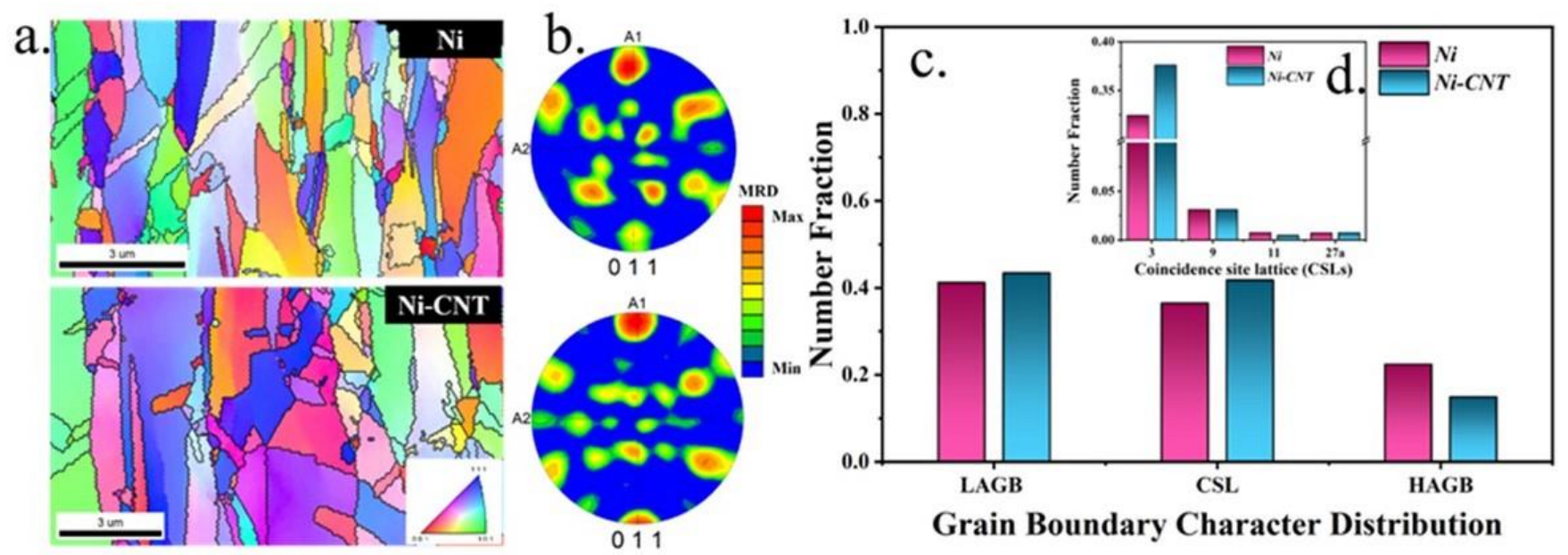

Figure 2. (a) Orientation distribution maps along the coating cross-section, (b) (011) pole figures, Histogram representing (c) Grain boundary character distribution, (d) Coincidence site lattice distribution in $\mathrm{Ni}$ and Ni-CNT coatings.

References

[1] M. K. Punith Kumar, M. P. Singh, C. Srivastava, RSC Adv. 5 (2015) p. 25603.

[2] Rekha M Y, C. Srivastava, Corros. Sci. 152 (2019) p. 234.

[3] M. K. Punith Kumar, Rekha M. Y., C. Srivastava, Corros. Rev. 39(1) (2021) p. 15. 